\section{Development of comprehension of logical connectives: Understanding of "or"*}

\author{
EDITH D. NEIMARK
}

Douglass College, Ruters-The State University, New Brunswick, N.J. 08903

A 16-item test for comprehension of set inclusion and exclusion, set intersect, and set union was administered to 162 high school students in Grades 9-12 for the purpose of assessing development of comprehension of "or" in this age range. Following completion of the first test, an alternate form was administered to provide additional information on sources of error in dealing with set union. Analysis of number of correct responses revealed that comprehension of "or" develops during the high school age range. Analysis of errors on set union questions suggested that (1) dealing correctly with set union requires formal operations, and (2) most errors result from inadequate applications of concrete operations.

Previous research in development of comprehension of logical connectives in Japanese (Nitta \& Nagano, 1966) and American (Neimark \& Slotnick, 1970) school children shows that, while comprehension of class inclusion or exclusion $(A, \bar{A})$ and class intersect $(A$ and $B)$ are present quite early, there is little comprehension of class union (A or B), even among eighth and ninth graders. The present experiment was conducted (1) to trace the development of understanding of logical connectives throughout the high school age range and (2) to shed additional light upon the reasons for the late appearance of comprehension of "or."

\section{MATERIALS}

Each $S$ was given one form of a 16-item test (Neimark \& Slotnick, 1970) which required him to circle all of the items described by the statement that preceded them. The first four statements dealt with class inclusion and exclusion: $A, \bar{A}, B, \bar{B}$; the next four statements involved intersection of classes: $A$ and $B, \bar{A}$ and $\bar{B}$, $A$ and $\bar{B}, \bar{A}$ and $B$; the remaining eight involved class union: $A$ or $B, \bar{A}$ or $\bar{B}, A$ or $\overline{\mathrm{B}}, \overline{\mathrm{A}}$ or $\mathrm{B}$. The first four union questions were phrased in the form "A or B or both" to clarify the inclusive interpretation of "or," while the last four used an unqualified "A or B."1 There were two equivalent forms of the test: one employed pictures as items, the other common nouns. The picture version employed as elements the eight pictures used by Nitta and Nagano in their Test 7: intersecting sets in which $A=$ birds, $\bar{A}=$ flowers, $\mathrm{B}=$ black, and $\overline{\mathrm{B}}=$ white. The eight elements were two black and two white

*This research was supported by Grant No. HD 1725-05 from the USPHS. We are grateful to Mr. John Sprout and the mathematics department of the Highland Park High School for their cooperation. The assistance of Thomas Ulrich in scoring and analysis of the data is gratefully acknowledged. birds and two black and two white flowers; the two members of each instance differed with respect to the irrelevant dimension of size. The word version was a modification of Nitta and Nagano Test 9, which, again, involved intersecting sets: $\mathbf{A}=$ flying things and $B=$ living things. The eight elements were Airplane, Bee, Warship, Goldfish, Kite, Sparrow, Tricycle, and Elephant. There were also two alternate forms of each version of the test. In one form, the matching form, there was a column of statements on the left and a column of encircled elements on the right. $S$ was to fill in the blank beside each statement with the letter of the correct answer from the column on the right. This form is, in essence, a recognition test version of the original test. In the second form 13 sets of encircled elements were presented with a blank beside each in which $S$ was to write his own description of the set of circled elements. The 13 sets consisted of the four inclusion and exclusion items, the four intersect items, the four union items, and one indescribable set, in a scrambled order. SUBJECTS

Intact algebra and senior math classes of the Highland Park High School served as Ss. These particular classes were chosen to achieve comparability with the 6 th-, 7 th-, and 8 th-grade math classes from Highland

Table 1

Composition of the Groups in Terms of Grade, Sex, Mean, and Standard Deviation of Chronological Age in Months, and Number of Subjects

\begin{tabular}{llcccr}
\hline & & \multicolumn{4}{c}{ Grade } \\
Sex & Measure & 9 & 10 & 11 & 12 \\
\hline Boys & CA, Mean & 183.28 & 189.06 & 201.35 & 207.75 \\
& SD & 9.05 & 5.68 & 5.87 & 6.48 \\
& N & 19 & 17 & 37 & 8 \\
Girls & CA, Mean & 179.08 & 190.56 & 198.51 & 209.33 \\
& SD & 8.62 & 6.50 & 7.89 & 4.47 \\
& N & 25 & 9 & 41 & 6 \\
Total & CA, Mean & 180.88 & 189.95 & 199.80 & 208.43 \\
& SD & 9.04 & 6.02 & 7.18 & 5.84 \\
& N & 44 & 26 & 78 & 14 \\
\hline
\end{tabular}

Park used in the earlier study. The composition of the groups with respect to chronological age in months and sex is summarized in Table 1.

\section{PROCEDURE}

The standard 16-item form in which $S$ was to circle all elements described by the statement to the left of it was distributed in an alternating fashion so that half the members of the class had the picture form while their neighbors had the verbal form. After the instructions were given and a sample problem involving geometric forms was done on the blackboard, $S$ was free to work at his own pace. Upon completion of the first form, $S$ raised his hand and $E$ replaced the completed test with one of the alternate forms (matching statements and answers, or describing each encircled set) involving elements he had not previously worked with: 79 Ss completed the matching form and 39 Ss completed the description form.

\section{RESULTS AND DISCUSSION}

Total Number Correct

Group mean number of correct answers in each question category of the standard form are plotted in Fig. 1 along with previous data from Neimark \& Slotnick (1970) for Grades 3.9 and college students. Data for pictorial elements are presented by solid lines and closed circles, for words in broken lines and open circles; in all cases, maximum number correct is four. As was expected on the basis of earlier findings, inclusion and intersect questions are correctly answered by high school students; for questions concerning set union, $\mathrm{A}$ or $\mathrm{B}$, on the other hand, there is a sharp increase in number correct from the 9 th-grade to the 12th-grade level for both the inclusive form of the question ("A or B or both," shown on the right) and unqualified form ("A or B," shown on the left). The assumption, based on earlier findings, that comprehension of "or" develops during the high school age range appears to be clearly supported.

On a priori analysis, the ability to perform unions of sets would seem to presuppose prior development of skills for 


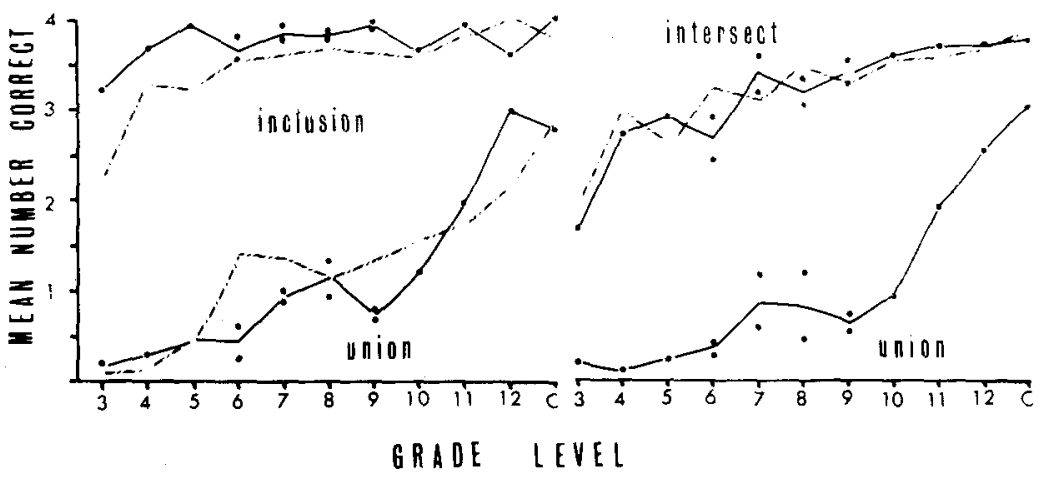

Fig. 1. Number of correct answers in each question category as a function of grade level. The union items on the left are unqualified, "A or $B$;" those to the right are the inclusive form, "A or B or both." Tests with pictorial elements are summarized by closed circles and solid lines; word elements are summarized by open circles and broken lines. Four is the maximum possible correct on each category.

(1) dealing with propositions and (2) generating all possible combinations of bivalued propositions. If this analysis is correct, then comprehension of "or" involves formal operations thinking and should be attained around the age of 15-16 when other cognitive abilities dependent upon formal operations appear, e.g., hypothetico-deductive reasoning (Inhelder \& Piaget, 1958) and generation of permutations (Leskow \& Smock, 1970). Although the present data are compatible with that interpretation, they could also be the result of an artifact of $S$ selection. Specifically, it is quite possible that poorer students drop out of school altogether and that only the brighter ones go on to take advanced algebra and senior math; if so, the older high school groups should contain a higher proportion of brighter students who would be expected to do better on the most difficult items. The best grounds for concluding that a developmental process is involved would be longitudinal data on the same $S$ s over a period of time. The present data provide a bit of longitudinal evidence: 13 of the 9 th-grade Ss had been tested a year before in the Neimark and Slotnick study. A comparison of performance change over a 1-year period for these $S$ s reveals a significant increase in the total number of correct responses: a $t$ for paired measures, with $12 \mathrm{df}$, of 2.39 is significant at the .05 level. For the most part, this improvement is attributable to an increase in the number of correct answers to "or" questions; where $S$ continues to make an error, it is generally the same error he made the previous year. Thus, it would appear that the improvement with age reflects a genuine process of cognitive development.

Previous data from younger Ss (Neimark \& Slotnick, 1970) had shown (1) better performance, especially at younger ages, with pictorial than with verbal elements and (2) a tendency toward more correct responses for the unqualified as compared with the inclusive form of set union items. With Ss at a transitional stage of understanding, one would expect that variation in question phrasing and nature of items might make for a difference in level of performance. For the present data these differences no longer obtain: (1) for pictorial vs verbal items $\mathrm{t}<1$ for all groups combined and for each grade separately; (2) for unqualified vs inclusive "or" questions, a $t$ for paired measures for the pictorial form is, again, not statistically significant. Thus, as Ss master the skills involved, their performance appears to become more consistent over variation in nature of material and phrasing of question.

In the first test, $\mathrm{S}$ had a descriptive statement and was to supply an answer by circling all the items to which it applied; in the second test, he was either given a set of statements and a set of answers to be matched up correctly (the matching test) or a set of encircled alternatives to each of which he was to supply a description (the description test). In relation to the initial standard form of the test, one might expect the matching test to be simpler and the description test to be more difficult;

this a priori assumption did not appear to be supported by the data. However, because of the experimental procedure employed. it is not possible to assess relative difficulty of the three test forms. In general. performance tended to be comparable on the first and second tests; this finding suggests that S's performance on the standard form was a valid and reliable measure of his understanding of logical connectives.

\section{ANALYSIS OF ERRORS}

Errors on all questions involving "or" are summarized in Table 2 with respect to three categories which accounted for most of the errors: Category 1 refers to interpreting set union (A or $B$ ) as set intersect ( $A$ and $B$ ), Category 2 to giving one or another of the component classes (generally the first) in place of their union (e.g., $\overline{\mathrm{A}}$ for $\overline{\mathrm{A}}$ or B), and Category 3 to an answer which is essentially correct, except that there are one too many or one too few encircled items. This type of error, which can occur only on the standard form, is probably attributable to carelessness. The first entry in each cell of Table 2 gives relative frequency of the error category in terms of percent of all responses, the second in terms of percent of all errors. Regardless of the form of the test or the nature of the items presented, one major source of error is to interpret "or" as "and" (Category 1); the relative frequency of occurrence of this type of error tends to decrease with age. Both of these findings replicate the results of prior work with younger Ss (Nitta \& Nagano, 1966; Neimark \& Slotnick, 1970).

Why does set union pose such difficulty even for high-school age children? The descriptions given on the description form of the test suggest part of the answer to be that children at these ages do not even use the word "or" spontaneously in this context (despite the fact that they have just completed a standard form of the test in which the word appears eight times). Only 6 of 19 Ss given the verbal form used "or" in their descriptions, while none of 20 Ss given the pictorial form did so; the

Table 2

Nature of Error as a Percent of All Responses and, in Parenthesis, as a Percent of All Errors

\begin{tabular}{|c|c|c|c|c|c|c|}
\hline \multirow[b]{2}{*}{ Group } & \multicolumn{3}{|c|}{ Pictures } & \multicolumn{3}{|c|}{ Words } \\
\hline & $I^{*}$ & II & III & I & II & III \\
\hline 9 Standard & $46.2(56.2)$ & $25.0(30.4)$ & $2.5(3.0)$ & $27.0(39.5)$ & $13.5(19.8)$ & $6.2(9.1)$ \\
\hline 9 Matching & $65.3(67.1)$ & $23.6(24.3)$ & - & $42.1(56.2)$ & $25.0(33.3)$ & -- \\
\hline 10 Standard & $44.9(64.3)$ & $10.6(15.1)$ & $2.6(3.8)$ & $26.4(35.9)$ & $15.3(20.8)$ & $8.3(11.3)$ \\
\hline 10 Matching & $50.0(55.6)$ & $25.0(27.8)$ & $\ldots$ & $31.2(43.5)$ & $25.0(34.8)$ & -- \\
\hline 11 Standard & $30.1(58.8)$ & $16.7(32.5)$ & $1.9(3.8)$ & $15.5(23.9)$ & $16.4(25.3)$ & $6.0(9.3)$ \\
\hline 11 Matching & $27.8(40.0)$ & $30.6(44.0)$ & $\ldots \ldots$ & $16.7(33.3)$ & $27.8(55.6)$ & $\ldots$ \\
\hline 11 Description & $4.8(16.0)$ & $1.2(4.0)$ & -- & $4.0(5.5)$ & $23.8(32.8)$ & -- \\
\hline 12 Standard & $3.6(14.3)$ & $14.3(57.2)$ & -- & $7.1(14.3)$ & $3.6(7.1)$ & $10.7(21.4)$ \\
\hline 12 Matching & $39.3(68.8)$ & $7.1(12.5)$ & -- & $7.1(66.7)$ & $3.6(33.3)$ & -- \\
\hline
\end{tabular}

"I refers to interpreting "or" as "and" (e.g., $A$ or $B=A$ and $B)$; II refers to interpreting union in terms of one or the other component set (e.g., $\bar{A}$ or $B=\bar{A}$ ); and III refers to probable carelessness. 
words "except" or "all but" were also infrequent (four Ss on the pictorial form). ${ }^{2}$ For the most part. Ss described set unions by compounding conjunctions, e.g., "all black not-birds and all birds." "black and not-black birds and not-black not-birds," "things that are not alive. are. and fly," "things that fly, are alive. not alive, and don't fly." The format of these descriptions suggests that $S$ has. in effect, a program for processing information that consists of two basic operations which are applied in series: casting into standard form (e.g., birds and not-birds) and scanning for properties. Thus, for example, in dealing with $\bar{A}$ or $\mathbf{B}$, after casting it into standard form, $\mathrm{S}$ scans for a property such as birdness, then for the second property (blackness), and reads out a summary of all included instances: all black birds and not-birds and all not-black not-birds. A very similar process appears to occur when $\mathrm{S}$ is given a description and must circle all included instances. The more operations the program includes the greater the likelihood of error, and error generally takes the form of stopping too soon, i.e., with an incomplete set of components, such as $\bar{A}, B, \bar{A}$ and $B, A$ and $B$, etc. Such an analysis is compatible with the error data of both the present and previous experiments.

Descriptions produced by the processing program outlined above are not only cumbersome but also ambiguous; anyone faced with the need for going through the process very often would be well advised to devise a more efficient and economical system, such as a system for dealing with unions as an identifiable entity. Apparently, late adolescents and adults have achieved such a system. But why should development of processing programs follow an apparent age-dependent sequence? One possible explanation is derivable from Piaget's treatment of cognitive development or variants of it (e.g., Neimark, 1970). Identification of properties is a development of early childhood (and is probably intimately related to language development); classification with respect to properties and even intersection of property classes is a concrete operation that is perfected during the 7-10 age range. Thus, it is not surprising that identification of sets appears first, followed by ability to deal with intersection of sets. When confronted with more complex tasks, such as dealing with disjunction, or set union, the child should employ operations already in his repertoire (such as constructing intersects of sets and set intersects). Ability to deal with more complex structures implies an ability to deal with all 16 binary combinations of classes; this is a formal operation and an attainment of adolescence. Thus. it is not surprising to find that (1) understanding of "or" develops over the high schoo! age range and (2) errors in dealing with "or" are largely attributable to application of concrete operations which, in turn, are inadequate for set unions.

\section{REFERENCES}

INHEIDER, B.. \& PIAGET, J. The growth of logical thinking from childhood to adolescence. New York: Basic Books, 1958.

LESKOW. S.. \& SMOCK. C. D. Developmental changes in problem solving stratevies: Permutation. Developmental Psychology, 1970. 2. 412-422.

NEMARK. F. D. Model for a thinking machine:
An information-processing framew ork for the study of cognitive development. Merrill-Palmer Quarterly, 1970.16 (in press).

NEMARK. E. D., \& SLOTXICK. $\& . S$ Development of the understanding of logical connectives. Journal of Iducational Psychology, 1970. in press.

VITA. N., \& NAGANO, S. Basic logical operations and their verbal expressions. Research Bulletin of the National Institute for Educational Research, 1966, No. 7.

$$
\text { NOTES }
$$

1. Due to a misprint on Question 9 of the standard verbal form. it was necessary to omit Items $9-12$ of this form from further analy sis.

2. One $S$, on the pictorial form, tried to get around the problem of appropriate connective by varying the quantifier. i.e., using "some" rather than "all" for set union descriptions.

\title{
The effect of starting pattern on descriptions of perceived temporal patterns*
}

\author{
DAVID PREUSSER, W. R. GARNER, and RICHARD L. GOTTWALD \\ Yale University, New Haven, Conn. 06510
}

Ss listened to and then described continually repeating sequences of nine tones presented at the rate of three tones/second. Each tone was either $275 \mathrm{~Hz}$ (high) or $250 \mathrm{~Hz}$ (low). The major independent variable was starting pattern (where $\mathrm{E}$ began the continually repeating sequence). The major dependent variable was S's description. The results showed that the "better" the starting pattern, the more often it was accepted and used as the description. "Better" starting patterns were also learned more quickly.

The temporal patterns used in the present study were fixed-length sequences of dichotomous tones (high vs low). When one of these patterns is continuously repeated to form a single ongoing sequence, we can distinguish between the starting pattern and the response or description pattern chosen by S. For instance, the continuing sequence

\section{H(high)L(low)HHHLLILHLHHH}

\section{LLLLHLHHHLL . .}

can be correctly described beginning at any point in the sequence, as HLHHHLLLL, LHHHLLLLH, HHHLLLLHL, etc., even though the starting pattern was HLHHHLLLL.

Two previous experiments have investigated the effects of starting pattern on S's pattern description. Royer \& Garner (1966) presented their Ss with continually repeating sequences of eight tones at the

*This research was supported by Grant YH 14229 from the National Institute of Mental Health to Yale University. The authors wish to thank Elinor Gamer for her help in data collection and tabulation. rate of two tones/second. They used all eight possible starting patterns. Their results showed only a negligible effect of starting pattern on pattern description. Garner \& Gottwald (1968) used patterns of eight tones or lights at rates of presentation from 0.8 to 8.0 events/second. Their results showed that the starting pattern was used as the response pattern if it provided a preferred, or frequently used, pattern description but was seldom used if the starting patterns provided a nonpreferred pattern description. This conclusion is limited, however, since for any given pattern only two of the eight possible starting patterns were used.

It is now known that $S s$ will describe a perceived temporal pattern with only those response patterns which do not break runs of identical tones (Royer \& Gamer, 1970). For instance, the pattern HLHHHLLLL . . will only be described as (coded in terms of run lengths) 1134 , 1341,3411 , or 4113 . Therefore, the present study used only those starting patterns which did not break a run. This selection yields, for four-run patterns, four starting patterns instead of the two or eight previously used. Further, these four starting patterns can be ordered in terms of 